ARTICLE

https://doi.org/10.1038/s41467-019-12677-6

\title{
Oligomeric state of the ZIKV E protein defines protective immune responses
}

Stefan W. Metz (1) ${ }^{1 \star}$, Ashlie Thomas ${ }^{1}$, Alex Brackbill², John Forsberg${ }^{2}$, Michael J. Miley², Cesar A. Lopez ${ }^{1}$ Helen M. Lazear (1) ${ }^{1}$, Shaomin $\operatorname{Tian}^{1} \&$ Aravinda M. de Silva ${ }^{1 \star}$

The current leading Zika vaccine candidates in clinical testing are based on live or killed virus platforms, which have safety issues, especially in pregnant women. Zika subunit vaccines, however, have shown poor performance in preclinical studies, most likely because the antigens tested do not display critical quaternary structure epitopes present on Zika E protein homodimers that cover the surface of the virus. Here, we produce stable recombinant $E$ protein homodimers that are recognized by strongly neutralizing Zika specific monoclonal antibodies. In mice, the dimeric antigen stimulate strongly neutralizing antibodies that target epitopes that are similar to epitopes recognized by human antibodies following natural Zika virus infection. The monomer antigen stimulates low levels of E-domain III targeting neutralizing antibodies. In a Zika challenge model, only E dimer antigen stimulates protective antibodies, not the monomer. These results highlight the importance of mimicking the highly structured flavivirus surface when designing subunit vaccines.

\footnotetext{
${ }^{1}$ Department of Microbiology and Immunology, University of North Carolina at Chapel Hill, Chapel Hill, NC, USA. ${ }^{2}$ Department of Pharmacology, University of North Carolina at Chapel Hill, Chapel Hill, NC, USA. *email: swmetz@med.unc.edu; aravinda_desilva@med.unc.edu
} 
Z ika virus (ZIKV) is an enveloped single stranded RNA virus that is transmitted by mosquito vectors. As a member of the flavivirus genus, ZIKV shares many structural features with other medically important flaviviruses such as Wes Nile virus, yellow fever virus, and dengue virus (DENV). The flavivirus envelope (E) protein is a major target of neutralizing and protective human antibodies. E oligomers form complex quaternary structure epitopes on the viral surface which are of particular importance as protective human antibodies bind to these higher order protein structures ${ }^{1}$.

ZIKV infection during pregnancy can result in neurodevelopmental defects (congenital Zika syndrome). This has stimulated work on subunit vaccines, as live attenuated and other replicating virus vaccines are contraindicated during pregnancy. However, for flaviviruses subunit vaccines based on recombinant $\mathrm{E}$ proteins (rE) have performed poorly in preclinical studies compared with virus or virus-like particle (VLP) vaccines ${ }^{2-6}$. In aqueous solution, flavivirus $\mathrm{rE}$ proteins are in a dynamic equilibrium that favors the monomer over the dimer at physiological temperature, which likely explains poor binding by potently neutralizing human antibodies targeting quaternary epitopes and the overall poor immunogenicity of these antigens in preclinical studies ${ }^{2,7,8}$.

Here, we investigate if ZIKV rE dimers are better subunit vaccine antigens than monomers. Unlike the $\mathrm{E}$ monomer, the dimer is recognized by strongly neutralizing mAbs isolated from ZIKV patients and the dimer, not the monomer, stimulates strongly neutralizing and protective antibodies that targeted epitopes that were similar to epitopes recognized by human antibodies following natural Zika virus infection. These results highlight the importance of mimicking the highly structured flavivirus surface when designing subunit vaccines. The flavivirus field has a long history of using $\mathrm{E}$ monomers as vaccine antigens with limited success. These results highlight the importance of mimicking the highly structured flavivirus surface when designing subunit vaccines, and are applicable to developing second generation subunit vaccines against Zika as well as other medically important flaviviruses like dengue and yellow fever.

\section{Results}

Expression of ZIKV rE monomers and homodimers. Here, to investigate if $\mathrm{ZIKV} \mathrm{rE}$ dimers are better subunit vaccine antigens than monomers, we expressed ZIKV rE variants (aa1-402) that are monomers $\left(\mathrm{rE}^{\mathrm{M}}\right)$ or stable dimers $\left(\mathrm{rE}^{\mathrm{D}}\right)$ under physiological conditions. We generated stable ZIKV $\mathrm{rE}^{\mathrm{D}}$ proteins as previously described for DENV $\mathrm{E}$ protein by introducing a disulfide bridge (A264C) in the E-domain II interactive region of the homodimer ${ }^{9,10}$ (Fig. 1a). The oligomeric state of the purified $\mathrm{rE}^{\mathrm{M}}$ and $\mathrm{rE}^{\mathrm{D}}$ proteins were confirmed by protein gel electrophoresis (Fig. 1b). The stable $\mathrm{rE}^{\mathrm{D}}$, but not the $\mathrm{rE}^{\mathrm{M}}$, was efficiently recognized by neutralizing $\mathrm{mAbs}$ that have dimer-dependent quaternary footprints, such as ZIKV-specific mAbs A9E, G9E, and ZKA-230 and flavivirus cross-reactive mAbs $\mathrm{C} 8$ and $\mathrm{C} 10$ (Fig. 1c).

ZIKV $\mathbf{r E}^{\mathrm{D}}$, but not $\mathbf{r E}^{\mathrm{M}}$, induces neutralizing antibodies. After confirming that $\mathrm{rE}^{\mathrm{D}}$ was a dimer displaying native quaternary epitopes, we immunized C57BL/6 mice with $5 \mu \mathrm{g} \mathrm{rE} \mathrm{M}^{\mathrm{M}}, 5 \mu \mathrm{g} \mathrm{rE}$,

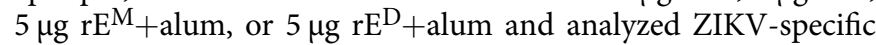
IgG and neutralizing antibody titers over time (Fig. 2a). In the absence of alum, $\mathrm{rE}^{\mathrm{M}}$ and $\mathrm{rE}^{\mathrm{D}}$ induced minimal IgG titers after the initial immunization, but this response increased upon boosting (Fig. 2b, c). In the alum adjuvanted groups, high IgG titers were observed even after the prime alone, with minimal change upon boosting (Fig. 2b). Although $\mathrm{rE}^{\mathrm{M}}$ elicited ZIKVbinding antibodies in the absence of alumn, these antibodies failed to neutralize ZIKV (Fig. 2d). In contrast, non-adjuvanted $\mathrm{rE}^{\mathrm{D}}$ elicited ZIKV-neutralizing antibodies (Fig. 2d). The addition of alum adjuvant elevated neutralizing antibody levels in both groups, but neutralizing titers were significantly higher after $\mathrm{rE}^{\mathrm{D}}$ immunization compared with $\mathrm{rE}^{\mathrm{M}}$. Our results demonstrate that, while both antigens stimulated ZIKV-specific IgG, the $\mathrm{rE}^{\mathrm{D}}$ stimulated antibodies that are more potently neutralizing compared with the $\mathrm{rE}^{\mathrm{M}}$ stimulated antibodies.

ZIKV rE $^{\mathrm{D}}$ stimulates antibodies that target complex epitopes. We next performed experiments to determine if antibodies stimulated by $\mathrm{rE}^{\mathrm{M}}$ and $\mathrm{rE}^{\mathrm{D}}$ antigens recognized different epitopes on the ZIKV E protein. Many flavivirus type-specific antibodies recognize relatively simple epitopes on domain III of the E protein (EDIII), whereas other type-specific antibodies recognize more complex quaternary structure epitopes displayed on E protein homodimers or higher order structures that cover the viral surface. Most EDIIIspecific neutralizing antibodies have been isolated from mice immunized with inactivated flavivirus antigens ${ }^{11-13}$. In contrast, most quaternary epitope directed neutralizing antibodies have been isolated from people infected with wild-type flaviviruses ${ }^{7,14-17}$. In fact, recent studies demonstrate that neutralizing antibodies in

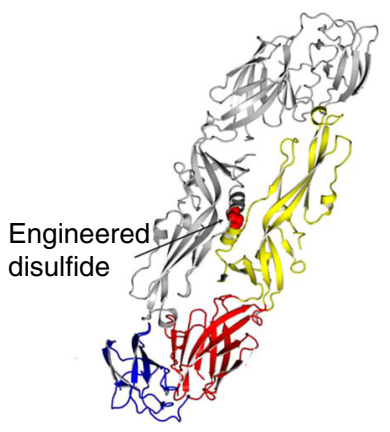

b

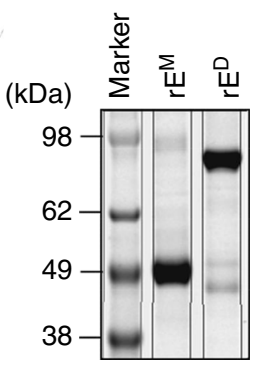

C

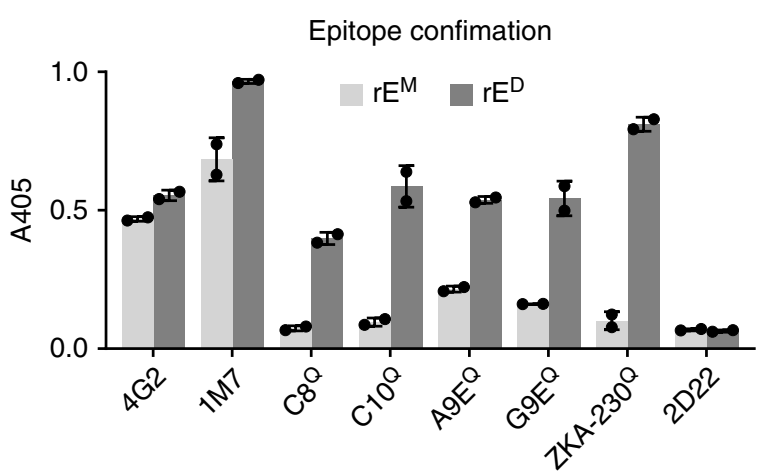

Fig. 1 Expression pf ZIKV rE monomers and rE homodimers. a The soluble ZIKV rE-monomer (rEM, aa1-404) was expressed and purified from mammalian cells. The soluble stable ZIKV rE-homodimer ( $\left.r E^{D}\right)$ was generated by an A264C substitution, resulting in a disulfide bridge in the E-domain II dimer interphase (yellow). b Purified $\mathrm{rE}^{\mathrm{M}}$ and $\mathrm{rE}^{\mathrm{D}}$ were analyzed by SDS-PAGE and exhibited predicted molecular weights of $\sim 49 \mathrm{kDa}$ (monomer) and $\sim 98 \mathrm{kDa}$ (dimer). c Binding of ZIKV specific (A9E, G9E, and ZKA-230) and flavivirus cross-reactive (4G2, 1M7, C8, and C10) mAbs to ZIKV rEM and rE ${ }^{D}$ was analyzed. Binding of $m A b s$ that have a quaternary footprint (Q) was only observed for $r E^{D}$. The DENV2 specific mAb $2 D 22$ was used as a negative control. Results from $n=2$ independent experiments are shown. Error bars represent standard deviation. Source data are provided as a Source Data file 
a

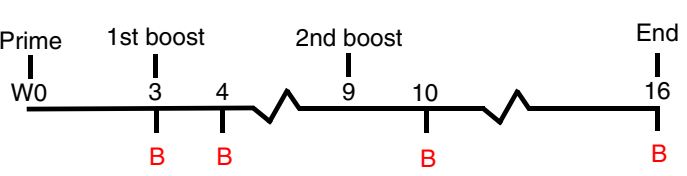

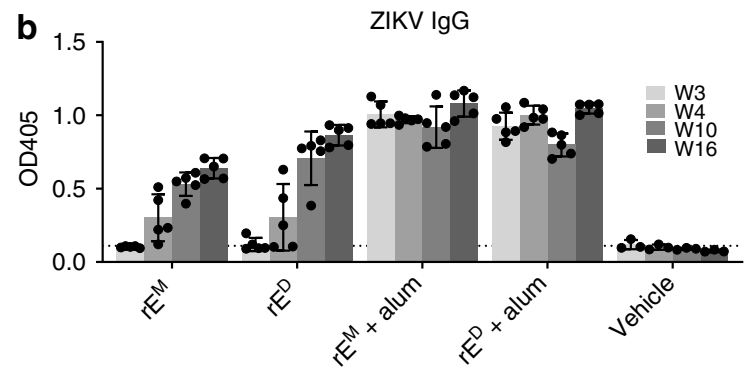

C

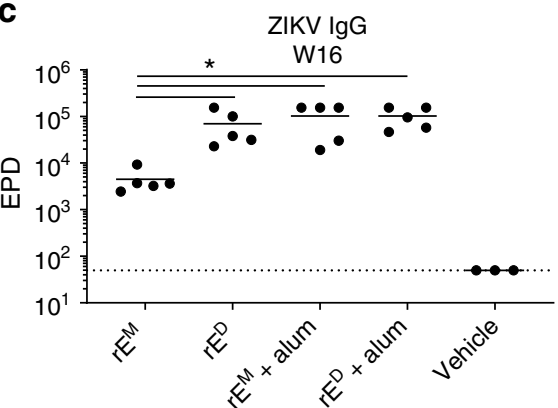

d

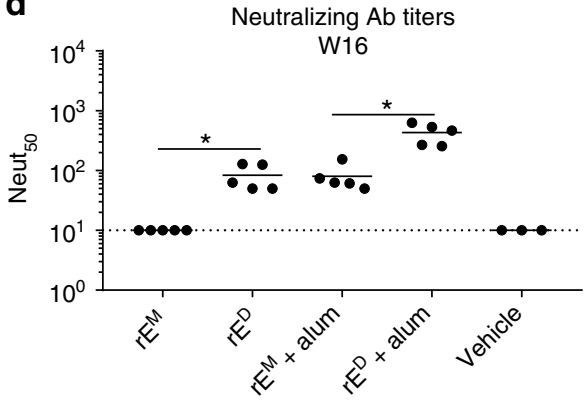

Fig. 2 ZIKV rED induces neutralizing antibody responses. a Mice were primed with $5 \mu$ g of ZIKV rEM or rED ( $+/-$ alum) and boosted with similar doses at weeks 3 and 9. Mice were bled (b) at indicated time points ( $n=5$ for $\mathrm{rEM}^{\mathrm{M}}, \mathrm{rE} \mathrm{E}^{\mathrm{D}}, \mathrm{rE} \mathrm{E}^{\mathrm{M}}+$ alum, and $\mathrm{rE}^{\mathrm{D}}+$ alum, and $n=3$ for vehicle control). $\mathbf{b} \mathrm{ZIKV}$-specific single point dilution (1:50) IgG titers were determined by virus capture ELISA. c ZIKV-specific endpoint dilution (EPD) titers were determined at week 16 by virus capture ELISA; EPD is the serum dilution at which detection signal equals background levels. $\mathbf{d}$ ZIKV-neutralizing activity at week 16 was determined on Vero cells and expressed as the serum dilution at which $50 \%$ of the virus was neutralized (Neut 50 ). Statistical differences were determined by one-way ANOVA followed by a Tukey's test $\left({ }^{\star} p<0.05\right)$. Data points represent individual mice. Error bars represent standard deviation. Source data are provided as a Source Data file

people exposed to flavivirus infections or effective live attenuated vaccines predominantly recognize quaternary epitopes, with simple EDIII epitopes accounting for only a minor fraction of neutralizing antibodies $^{18-22}$. To compare levels of EDIII directed antibodies in animals immunized with $\mathrm{rE}^{\mathrm{M}}$ or $\mathrm{rE}^{\mathrm{D}}$, we used recombinant $\mathrm{ZIKV}$ EDIII conjugated to magnetic beads to deplete mouse immune sera of EDIII-binding antibodies (Fig. 3a). We tested control-depleted and EDIII antibody depleted immune sera to estimate the levels of binding (Fig. 3b) and neutralizing antibodies (Fig. 3c) in each sample targeting epitopes on EDIII. In mice immunized with $\mathrm{rE}^{\mathrm{M}}$, $\sim 70 \%$ ( $\mathrm{rE}^{\mathrm{M}}$ group) and $\sim 60 \%$ ( $\mathrm{rE}^{\mathrm{M}}+$ alum group) of total $\mathrm{ZIKV}$ specific IgG recognized epitopes on EDIII (Fig. 3b). On the other hand, significantly less EDIII-specific antibodies were induced after immunization with $\mathrm{rE}^{\mathrm{D}}\left(\sim 30 \%\right.$ for $\mathrm{rE}^{\mathrm{D}}$ and $\sim 20 \%$ for $\mathrm{rE}^{\mathrm{D}}+$ alum). The antibody binding patterns were also reflected in the ZIKV neutralization assays. The majority of neutralizing antibodies in the $\mathrm{rE}^{\mathrm{M}}+$ alum group were lost following depletion with EDIII antigen (Fig. 3c). In contrast, no loss of neutralization was observed in the animals immunized with $\mathrm{rE}^{\mathrm{D}}$ or $\mathrm{rE}^{\mathrm{D}}+$ alum after the removal of EDIII-binding antibodies (Fig. 3c). We conclude that the $\mathrm{rE}^{\mathrm{M}}$ antigen induces an EDIII focused antibody response that is poorly neutralizing, whereas $\mathrm{rE}^{\mathrm{D}}$ antigens stimulate robust neutralizing antibody responses that target epitopes distinct from simple EDIII epitopes.

We recently isolated two ZIKV-specific strongly neutralizing human mAbs (A9E and G9E) from a person who was infected with ZIKV in $2016^{23}$. These antibodies map to complex epitopes on domain I (A9E) and domain II (G9E) of ZIKV E protein ${ }^{23}$. Moreover, antibody blockade assays using these mAbs and human immune sera indicated that the epitopes targeted by these $\mathrm{mAbs}$ also are targeted by antibodies in immune serum from Zika patients but not dengue patients ${ }^{23}$. We used a blockade of binding (BOB) assay to test if mice immunized with $\mathrm{rE}^{\mathrm{M}}$ or $\mathrm{rE}^{\mathrm{D}}$ developed antibodies that recognize A9E or G9E epitopes on ZIKV. We also tested if $\mathrm{rE}^{\mathrm{M}}$ or $\mathrm{rE}^{\mathrm{D}}$ antigens stimulated antibodies that targeted the epitope of human mAb EDE C10 ${ }^{17}$ which binds to a $\mathrm{E}$ dimer-dependent epitope that is conserved between DENV and ZIKV. Sera from mice immunized with $\mathrm{rE}^{\mathrm{M}}$ did not block binding of A9E, G9E, and C10 mAbs to ZIKV (Fig. 3d). In contrast, sera from mice immunized with $\mathrm{rE}^{\mathrm{D}}$ antigen efficiently blocked binding of A9E and G9E ( 80\%) and partially blocked binding of EDE C10 ( 35\%) to ZIKV. These results demonstrate that the $\mathrm{rE}^{\mathrm{D}}$ antigen and natural ZIKV infections stimulate antibodies that target similar complex epitopes on the virion. The $\mathrm{rE}^{\mathrm{M}}$ fails to induce similar antibodies and directs the antibody response to simple epitopes on ZIKV EDIII.

ZIKV $\mathrm{rE}^{\mathrm{D}}$ induces protective antibodies in mice. Next, we investigated if the immune sera of mice vaccinated with $\mathrm{rE}^{\mathrm{M}}$ + alum and $\mathrm{rE}^{\mathrm{D}}+$ alum could protect mice from lethal ZIKV challenge. We immunized wild-type mice as previously described (Fig. 2), and transferred heat-inactivated immune sera to Ifnar1 $1^{-1-}$ mice which are highly susceptible to ZIKV. Ifnar1 $1^{-1-}$ mice were challenged with $1000 \mathrm{FFU}$ of ZIKV H/PF/2013, 1 day post serum transfer. Mice were monitored for weight loss and ZIKV induced disease, and mice were bled at days 0 (before virus challenge), 4, and 14 (Fig. 4a). The potently neutralizing mAb G9E was used as a positive control for protection against ZIKV infection ${ }^{23}$. Mice that received G9E or serum from $\mathrm{rE}^{\mathrm{D}}$ +alum immunization were completely protected against weight loss and disease signs. In contrast, mice receiving $\mathrm{rE}^{\mathrm{M}}+$ alum serum exhibited significant weight loss and hindlimb paralysis (Fig. 4b). At 4 days post challenge, viremia levels in the $\mathrm{rE}^{\mathrm{D}}$ + alum group were significantly reduced compared with the $\mathrm{rE}^{\mathrm{M}}$ +alum and vehicle groups (Fig. 4c), and all groups cleared viremia at day 14 . We also measured ZIKV-specific IgG titers (Fig. 4d) and neutralizing antibody titers (Fig. 4e) at days 0 and 14. Mice receiving $\mathrm{rE}^{\mathrm{M}}+$ alum and $\mathrm{rE}^{\mathrm{D}}+$ alum immune sera showed similar ZIKV-specific IgG titers at day 0, which were 
a

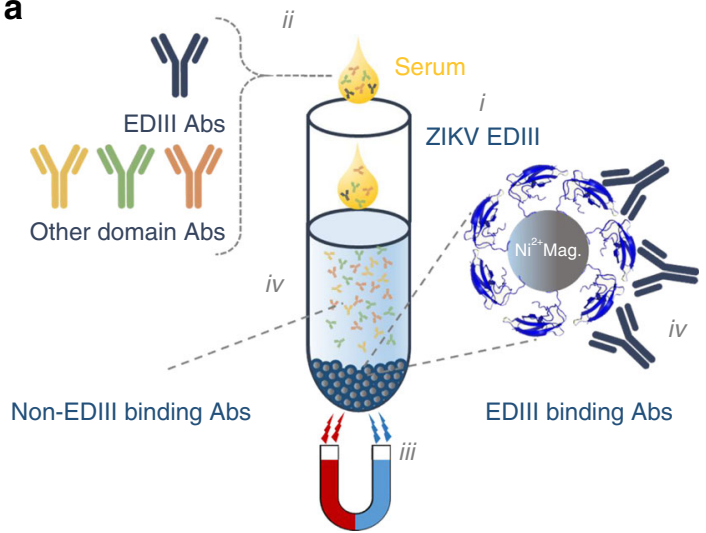

C

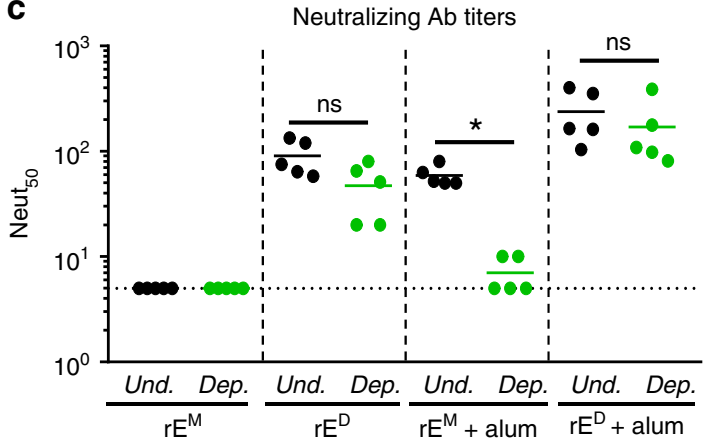

b

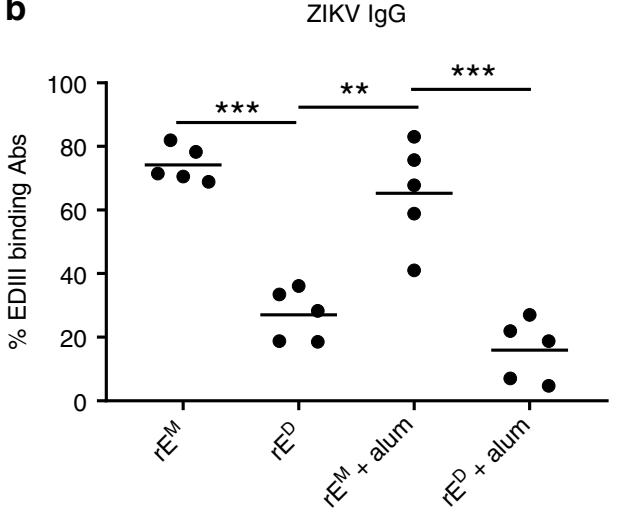

d

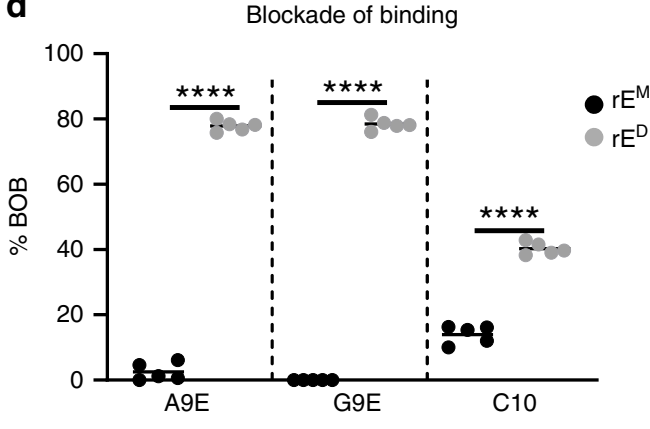

Fig. 3 ZIKV rED stimulates antibodies that target complex epitopes on the virion. a Method for depleting EDIII-binding antibodies from mouse immune sera. Recombinant ZIKV E-domain III (EDIII, His-tagged) was coupled to nickel beadsi then incubated with immune seraii. Magnetic pull down removes nickel

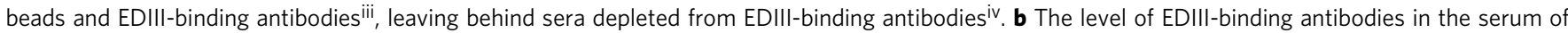
mice immunized with the indicated antigens is shown as a percentage of the total level of ZIKV-specific lgG, as measured by ELISA of EDIII-depleted and control-depleted serum. c The neutralizing activity of EDIII-depleted (green) and undepleted sera (black) was determined and expressed as the dilution at which $50 \%$ of the virus was neutralized (Neut ${ }_{50}$ ). d A blockade of binding (BOB) assay was used to evaluate if mice immunized with $\mathrm{rE}^{\mathrm{M}}$ (black) or $\mathrm{rE}^{\mathrm{D}}$ (gray) developed antibodies that blocked the binding of A9E, G9E, and EDE C10 human mAbs. Data points represent individual mice. Statistical differences were determined by one-way ANOVA followed by a Tukey's test $\left({ }^{\star} p<0.05\right)$. Source data are provided as a Source Data file

marginally higher for both groups at day 14 . On the other hand, neutralizing antibody titers were higher at day 14 in the $\mathrm{rE}^{\mathrm{M}}$ +alum group, likely caused by an endogenous immune response elicited against replicating virus in these animals (Fig. 4c).

\section{Discussion}

The magnitude, sudden onset and severe clinical manifestations of ZIKV epidemics highlight the need for safe and effective vaccines for key patient populations including pregnant women. While effective live attenuated vaccines have been developed for several flaviviruses, they are contraindicated in pregnant women. Live vaccines typically are ineffective during the first year of life because maternal antibodies interfere with vaccine replication. It has been difficult to formulate tetravalent live attenuated DENV vaccines that stimulate balanced and effective responses to each serotype because of differential replication of vaccine virus components. Subunit vaccines may address some of these challenges, but progress has been slow and subunit vaccines have not performed well in non-human primate models ${ }^{24}$. Our studies offer a potential explanation for the poor performance of subunit vaccines and a strategy for developing more effective flavivirus vaccines. Our results demonstrate that a critical aspect of $\mathrm{rE}$ antigen design is to develop $\mathrm{E}$ proteins that display complex quaternary epitopes that recapitulate antigen presentation on the surface of infectious virus particles. Here we demonstrate that $\mathrm{ZIKV} \mathrm{rE}^{\mathrm{M}}$ and $\mathrm{rE}^{\mathrm{D}}$ subunit antigens induce antibody responses that differ in functionality and domain focus. ZIKV rE monomers stimulated an EDIII biased, poorly neutralizing IgG response. In contrast, ZIKV rE dimers induced strongly neutralizing and protective antibodies that also blocked the binding of strongly neutralizing human mAbs to quaternary epitopes on ZIKV. While finer mapping studies are required to comprehensively map the specificity of $\mathrm{rE}^{\mathrm{D}}$ stimulated antibodies, our studies suggest that by designing the E protein to be a stable homodimer, it is possible to induce strongly neutralizing and protective antibodies that mimic properties of protective antibodies induced by natural infection. It is possible that inactivated whole virus or virus-like particle (VLP) vaccines will also stimulate antibodies to quaternary structure epitopes. An advantage to using $\mathrm{rE}^{\mathrm{D}}$-protein subunits over inactivated virion or VLP vaccines is the absence or prM protein in subunit vaccine but not virus or VLP vaccines. PrM antibodies are non-neutralizing and some studies suggest that enhance the replication of flaviviruses ${ }^{25}$. Additional studies are needed to compare the immunogenicity, efficacy and safety of ZIKV rE-dimer, inactivated virus and VLP vaccines. The strategy we describe here for ZIKV E protein may also be applicable for developing subunit vaccines against other medically significant flaviviruses.

\section{Methods}

Cells and viruses. Vero-81 cells (ATCC CCL-81) were maintained in DMEM medium (Gibco) $+1 \%$ non-essential amino acids, $5 \%$ fetal bovine serum, streptomycin $\left(100 \mu \mathrm{g}\right.$ per ml), and penicillin $\left(100 \mathrm{U}\right.$ per ml) at $37^{\circ} \mathrm{C}$ with $5 \% \mathrm{CO}_{2}$. 
a

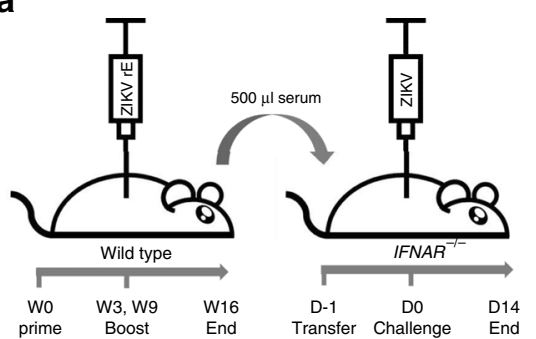

b

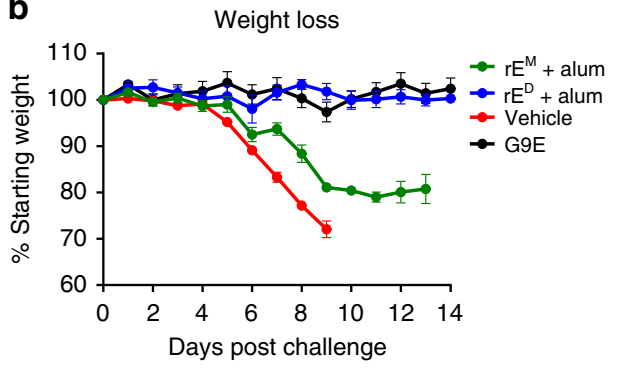

C

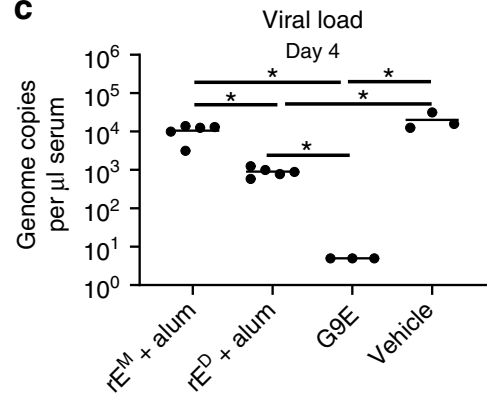

d

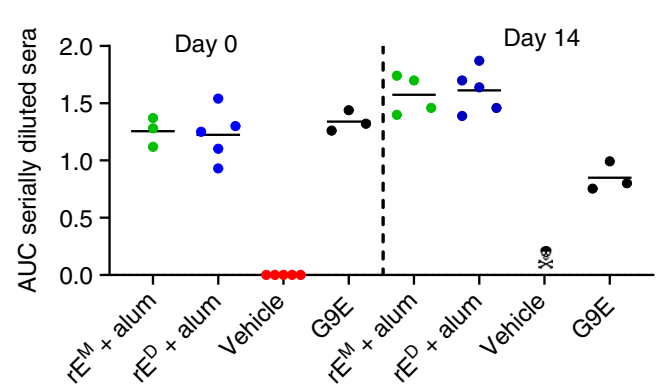

e

Neutralizing antibody titers

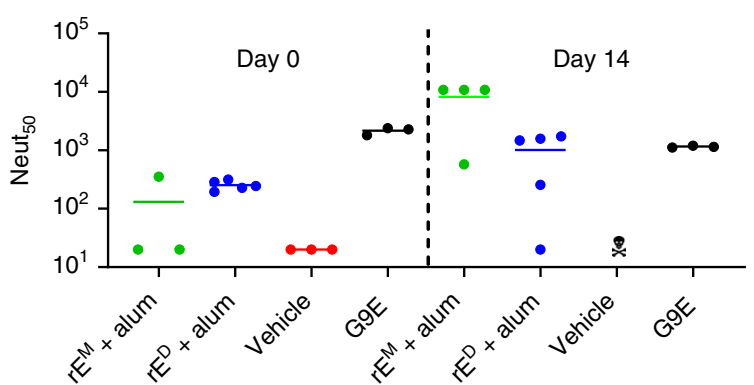

Fig. 4 ZIKV rED induce protective antibodies in mice. a Wild-type mice were immunized with $r E^{M}+$ alum or $r E^{D}+$ alum. Five hundred microliters of heatinactivated immune sera (16 weeks post immunization) (or $200 \mu \mathrm{g}$ of neutralizing $\mathrm{mAb}$ G9E) was transferred by intraperitoneal injection to Ifnar ${ }^{-/-}$mice $24 \mathrm{~h}$ prior to challenge with $1000 \mathrm{FFU}$ of ZIKV by subcutaneous footpad inoculation ( $n=5$ for $\mathrm{rEM}^{\mathrm{M}}$ and rED and $n=3$ for controls). $\mathbf{b}$ Challenged mice were weighed daily for 14 days; weights are shown percent of starting weight. Data represent the mean $+/-S E M$ of 3-5 mice per group and were censored once one mouse in a group died. c Viremia was measured at 4 days post challenge by qRT-PCR. d-e ZIKV-specific IgG and neutralizing antibody titers were determined for challenged mice prior to challenge (day 0 ) and at the end of the experiment (day 14 ). Statistical differences were determined by one-way ANOVA followed by a Tukey's test $\left({ }^{\star} p<0.05\right)$. Source data are provided as a Source Data file

Suspension adapted EXPI293 cells (ThermoFisher) were cultured in EXPI293 expression medium (Life Technologies). ZIKV strain H/PF/2013 was grown in Vero cells and titered by focus-forming assay using flavivirus mAb E6026,27.

Production of ZIKV rE antigens. The recombinant ZIKV E (H/PF/2013; aa1-404) monomers $\left(\mathrm{rE}^{\mathrm{M}}\right)$ and stable $\mathrm{E}$ dimers $\left(\mathrm{rE}^{\mathrm{D}}\right)$ were expressed in the EXPI293 transient expression system (ThermoFisher) following supplied protocols. The $\mathrm{rE}^{\mathrm{D}}$ was created by an A264C substitution in E-domain II and all proteins were equipped with a C-terminal $6 \times$ His-tag. Recombinant proteins were purified as previously described ${ }^{28}$. In short, cell supernatants were concentrated by tangential filtration and proteins were affinity purified using $\mathrm{Ni}^{2+}$-chromatography columns. Pooled elution fractions were subjected to size exclusion chromatography and purified protein samples were flash frozen and stored at $-80{ }^{\circ} \mathrm{C}$.

Production of ZIKV EDIII proteins. ZIKA virus EDIII (H/PF/2013, aa302-403) was expressed as an N-terminal MBP fusion protein equipped with a C-terminal $6 \times$ His-tag in BL21-E. coli. Cells were transformed with a pET21-MBP/ZIKVEDIII expression plasmid and expanded overnight to $100 \mathrm{ml}$ cultures (LB-growth medium, supplemented with $100 \mu \mathrm{g}$ per $\mathrm{ml}$ ampicillin). Overnight cultures were transferred to $1 \mathrm{~L} \mathrm{LB}$-growth medium and grown to and $\mathrm{OD}_{600}=0.6$ at $37^{\circ} \mathrm{C}$ shaking at $225 \mathrm{rpm}$. ZIKV EDIII expression was stimulated by adding $50 \mathrm{mM}$ IPTG and cells were grown overnight at $16^{\circ} \mathrm{C}$ with shaking at $225 \mathrm{rpm}$. Next, cells were pelleted and lysed by high pressure homogenization. ZIKV EDIII proteins were purified from the lysate by $\mathrm{Ni}^{2+}$-affinity chromatography. Eluted fractions (50 mM Tris, $10 \mathrm{mM} \mathrm{NaCl}, 10 \%$ glycerol, $10 \%$ sucrose, $\mathrm{pH} \mathrm{7.0)}$ ) were pooled and dialyzed overnight at $4{ }^{\circ} \mathrm{C}$ in $20 \mathrm{mM}$ Tris, $50 \mathrm{mM} \mathrm{NaCl}, \mathrm{pH} 7.0$ and stored at $-80^{\circ} \mathrm{C}$.

Protein analysis. The oligomeric state of purified $\mathrm{rE}^{\mathrm{M}}$ and $\mathrm{rE}^{\mathrm{D}}$ proteins was characterized by ELISA and SDS-PAGE followed by Coomassie Brilliant Blue (CBB) staining. For SDS-PAGE, $1 \mu \mathrm{g}$ of $\mathrm{rE}^{\mathrm{M}}$ and $\mathrm{rE}^{\mathrm{D}}$ were incubated in denaturing gel loading buffer for $10 \mathrm{~min}$ at $95^{\circ} \mathrm{C}$. After centrifugation, proteins were separated by SDS-PAGE and gels (original gel in Source Data File) were incubated with CBB stain ( $0.1 \%$ coomassie blue, $10 \%$ acetic acid, $50 \%$ methanol). Protein confirmations were analyzed by antigen-capture ELISA. ZIKV $\mathrm{rE}^{\mathrm{M}}$ and $\mathrm{rE}^{\mathrm{D}}$ were captured on $\mathrm{Ni}^{2+}$-coated ELISA plates and subjected to binding of ZIKV-specific 2 ng per $\mu \mathrm{l} \mathrm{mAbs}$ (A9E, G9E, and ZKA-230) ${ }^{23}$ and flavivirus cross-reactive mAbs $(4 \mathrm{G} 2 \text { and } 1 \mathrm{M} 7)^{16,29}$.
Mouse immunizations and challenge. Female C57BL/6 mice were purchased from Jackson Laboratory and used for immunizations at 6-12 weeks of age.

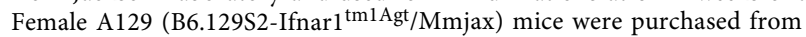
MMRRC (Mutant Mouse Resource \& Research centers supported by NIH) and used in challenge experiments at 7 weeks of age. All mouse experiments were performed under protocols approved by the University of North Carolina Institutional Animal Care and Use Committee, in compliance with ethical and federal regulations (the Public Health Service Policy on Humane Care and Use of Laboratory Animals, Animal Welfare Act, and the Guide for the Care and Use of Laboratory Animals).

For evaluation of ZIKV rE immunogenicity, mice were immunized subcutaneously in the flank with $5 \mu \mathrm{g}$ soluble $\mathrm{rE}^{\mathrm{M}}(n=5), 5 \mu \mathrm{g} \mathrm{rE}$ (Alhydrogel, Invivogen) $(n=5), 5 \mu \mathrm{g} \mathrm{rE} \mathrm{r}^{\mathrm{D}}(n=5), 5 \mu \mathrm{g} \mathrm{rE} \mathrm{r}^{\mathrm{D}}+500 \mu \mathrm{g}$ alum $(n=5)$, or PBS $(n=3)$. All groups received three immunizations (day 0,21 , and 63 ), and serum samples were collected on day $21,28,70,77,84,91,98,105$, and 112 by submandibular bleed.

To evaluate the protective activity of $\mathrm{rE}$-induced antibodies, heat-inactivated immune sera were passively transferred to ZIKV susceptible A129 mice. Serum samples (days 70 through 112) of mice immunized with $\mathrm{rE}^{\mathrm{M}}+$ alum and $\mathrm{rE}^{\mathrm{D}}+$ alum (days 70-112) were pooled and $500 \mu \mathrm{l}$ of immune sera (per mouse, $n=5$ ), or vehicle control serum $(n=3)$ was injected into A129 mice, via intraperitoneal route 1 day prior to ZIKV challenge. The ZIKV-neutralizing $\mathrm{mAb} G 9 \mathrm{E}^{23}$ was used as a positive control ( $200 \mu \mathrm{g} /$ mouse, $n=3)$. Mice were challenged with $1000 \mathrm{FFU}$ of ZIKV (H/PF/2013) by subcutaneous footpad inoculation. Mice were weighed daily for 14 days and monitored for clinical disease signs. Mice losing $30 \%$ of their starting weight were humanely euthanized.

ZIKV rE-induced antibody evaluation. ZIKV-specific IgG responses were measured by antigen-capture ELISA. Briefly, ZIKV was captured on 1M7 ( $2 \mathrm{ng}$ per $\mu$ l)coated ELISA plates and incubated with 1:50 or serially diluted mouse sera 3, 4, 10, and 16 weeks post immunization. Next, plates were incubated with alkaline phosphatase conjugated anti-mouse IgG (1:2500; Sigma A9044) diluted anti-mouse IgG for $1 \mathrm{~h}$ at $37^{\circ} \mathrm{C}$. Wells were developed using alkaline phosphatase substrate and adsorbance was measured at $405 \mathrm{~nm}$. Endpoint dilution (EPD) titers where mice sera signals reached background (vehicle control) levels was determined using GraphPad Prism software.

Evaluation of neutralizing antibody responses. Neutralizing antibody titers were determined using a flow cytometry based assay. Vero- 81 cells were seeded $(25,000$ cells per well) and incubated at $37^{\circ} \mathrm{C}$ overnight. Immunized mice sera was serially 
diluted in OptiMEM (Gibco) supplemented with 2\% FBS and incubated for 45 min at $37^{\circ} \mathrm{C}$ with the amount of ZIKV previously determined to infect $\sim 15 \%$ of the cells. Cells were subsequently washed with OptiMEM and overlaid with the diluted sera/virus mix for $2 \mathrm{~h}$ at $37^{\circ} \mathrm{C}$. Next, the cells were washed with growth medium and incubated overnight in growth medium at $37^{\circ} \mathrm{C}$. The next day, cells were washed with PBS and detached from the plate by trypsin (Gibco). Cells were subsequently fixed in $4 \%$ paraformaldehyde and washed in permeabilization buffer and blocked with $1 \%$ normal mouse serum in perm buffer for $30 \mathrm{~min}$ at room temperature. Following incubation, infected cells were detected by staining with Alexa-488 conjugated $1 \mathrm{M} 7 \mathrm{mAb}(5 \mu \mathrm{g}$ per $\mu \mathrm{l})$ for $1 \mathrm{~h}$ at $37^{\circ} \mathrm{C}$. Next, cells were washed in perm buffer and resuspended in $200 \mu \mathrm{l}$ FACS buffer. The percentage of infected cells were determined using the Guava Flow Cytometer (EMD Millipore) and the neutralizing capacity was expressed as neut ${ }_{50}$ (the dilution where $50 \%$ of the virus is neutralized), calculated by GraphPad Prism software.

Depletion of EDIII-binding IgG from mouse serum. Bacterially expressed ZIKV EDIII proteins (C-terminal $6 \times$ His-tag) were conjugated to Ni-NTA Magnetic beads (Thermo Scientific) following manufacturers protocol. In short, $1 \mathrm{mg}$ of Ni-NTA beads were washed in equilibration buffer and incubated on a rotator with $60 \mu \mathrm{g}$ of ZIKV-EDIII or MBP-His (control depletions) for $1 \mathrm{~h}$ at $37^{\circ} \mathrm{C}$. Next, the beads were washed in equilibration buffer and divided in two tubes for two rounds of depletions. Mouse immune serum was diluted 1:20 in PBS and incubated with magnetic beads for $1 \mathrm{~h}$ at $37^{\circ} \mathrm{C}$ on a rotator. Following incubation, the depleted serum was separated from the beads and stored at $4{ }^{\circ} \mathrm{C}$ for subsequent IgG ELISA and neutralization assays. The percentage of ZIKV EDIIIbinding antibodies was calculated by dividing the EDIII-depleted IgG titers by the control-depleted IgG titers. The percentage of ZIKV EDIII neutralizing antibodies was calculated by dividing the EDIII-depleted neut ${ }_{50}$ by the controldepleted neut ${ }_{50}$.

Human mAb blockade of binding (BOB) assay. High-binding ELISA plates were coated with $2 \mathrm{ng}$ per $\mu \mathrm{l} 1 \mathrm{M} 7 \mathrm{human} \mathrm{mAb}$ in PBS for $1 \mathrm{~h}$ at $37^{\circ} \mathrm{C}$. Next, plates were blocked with $3 \%$ skim milk in PBS $+0.05 \%$ Tween for $30 \mathrm{~min}$ at $37^{\circ} \mathrm{C}$. After blocking, plates were washed in PBS $+0.2 \%$ Tween and incubated with ZIKV $\mathrm{H} / \mathrm{PF} / 2013$ for $1 \mathrm{~h}$ at $37^{\circ} \mathrm{C}$. The plates were washed and incubated with serially diluted immunized mouse sera for $1 \mathrm{~h}$ at $37^{\circ} \mathrm{C}$. Next, the plates were washed and incubated with alkaline phosphatase (AP)-conjugated A9E, G9E or C10 human $\mathrm{mAb}$ at $2 \mathrm{ng}$ per $\mu \mathrm{l}$ for $1 \mathrm{~h}$ at $37^{\circ} \mathrm{C}$. Following incubation, the plates were washed and developed with AP-substrate and absorbance was measured at $405 \mathrm{~nm}$.

ZIKV RNA qRT-PCR analysis. Viral RNA was isolated from $30 \mu \mathrm{l}$ of mouse sera using the QIAmp Viral RNA Mini Kit (Qiagen) following manufacturer's protocol. RNA was eluted in RNase free MilliQ water and stored at $-80^{\circ} \mathrm{C}$. For qRT-PCR, 2 $\mu \mathrm{l}$ of RNA was added to $18 \mu \mathrm{l}$ of PCR mastermix ( $10 \mu \mathrm{l}$ of iTaq universal probes mix $+0.5 \mu \mathrm{l}$ of iScript advanced reverse transcriptase $+2 \mu \mathrm{l}$ of forward primer $(10$ $\mu \mathrm{M}$; CATGATACTGCTGATTGC $)+2 \mu \mathrm{l}$ of reverse primer $(10 \mu \mathrm{M}$; CCTTCCACAAAGTCCCTATTGC) $+0.4 \mu \mathrm{l}$ of probe (IDT; $10 \mu \mathrm{M}$; FAM-CGGCATACAZEN-GCATCAGGTGCATAGGAG-IBFQ) $+6.5 \mu \mathrm{l}$ of nuclease-free $\mathrm{H}_{2} \mathrm{O}$ ). Next, samples were analyzed in a Bio-Rad CFX96 Thermocycler according to the following program: $10 \mathrm{~min}$ at $50^{\circ} \mathrm{C}, 2 \mathrm{~min}$ at $95^{\circ} \mathrm{C}\left(10 \mathrm{~s}\right.$ at $95^{\circ} \mathrm{C}, 20 \mathrm{~s}$ at $\left.60^{\circ} \mathrm{C}\right) \times 40$ cycles. RNA was isolated from a known quantity of ZIKV H/PF/2013 to generate a standard curve.

Reporting summary. Further information on research design is available in the Nature Research Reporting Summary linked to this article.

\section{Data availability}

The authors declare that all data supporting the findings of this study are available within the paper. The source data underlying the figures are provided as a Source Data file.

Received: 10 June 2019; Accepted: 25 September 2019;

Published online: 10 October 2019

\section{References}

1. Gallichotte, E. N., Baric, R. S. \& de Silva, A. M. The molecular specificity of the human antibody response to dengue virus infections. Adv. Exp. Med. Biol. 1062, 63-76 (2018).

2. Larocca, R. A. et al. Vaccine protection against Zika virus from Brazil. Nature 536, 474-478 (2016).

3. Block, O. K. et al. A tetravalent recombinant dengue domain III protein vaccine stimulates neutralizing and enhancing antibodies in mice. Vaccine $\mathbf{2 8}$, 8085-8094 (2010).

4. Midgley, C. M. et al. An in-depth analysis of original antigenic sin in dengue virus infection. J. Virol. 85, 410-421 (2011).
5. Williams, K. L., Wahala, W. M., Orozco, S., de Silva, A. M. \& Harris, E. Antibodies targeting dengue virus envelope domain III are not required for serotype-specific protection or prevention of enhancement in vivo. Virology 429, 12-20 (2012).

6. Liu, Y., Liu, J. \& Cheng, G. Vaccines and immunization strategies for dengue prevention. Emerg. Microbes Infect. 5, 77-83 (2016).

7. de Alwis, R. et al. Identification of human neutralizing antibodies that bind to complex epitopes on dengue virions. Proc. Natl Acad. Sci. USA 109, 7439-7444 (2012).

8. Allison, S. L., Stadler, K., Mandl, C. W., Kunz, C. \& Heinz, F. X. Synthesis and secretion of recombinant tick-borne encephalitis virus protein $\mathrm{E}$ in soluble and particulate form. J. Virol. 69, 5816-5820 (1995).

9. Slon Campos, J. L. et al. Temperature-dependent folding allows stable dimerization of secretory and virus-associated E proteins of Dengue and Zika viruses in mammalian cells. Sci. Rep. 7, 966 (2017).

10. Rouvinski, A. et al. Covalently linked dengue virus envelope glycoprotein dimers reduce exposure of the immunodominant fusion loop epitope. Nat. Commun. 8, 15411 (2017).

11. Wahala, W. M. et al. Natural strain variation and antibody neutralization of dengue serotype 3 viruses. PLoS Pathog. 6, el000821 (2010).

12. Pitcher, T. J. et al. Functional analysis of dengue virus (DENV) type 2 envelope protein domain 3 type-specific and DENV complex-reactive critical epitope residues. J. Gen. Virol. 96, 288-293 (2015).

13. Zhou, Y. et al. The mechanism of differential neutralization of dengue serotype 3 strains by monoclonal antibody 8A1. Virology 439, 57-64 (2013).

14. Fibriansah, G. et al. DENGUE VIRUS. Cryo-EM structure of an antibody that neutralizes dengue virus type 2 by locking E protein dimers. Science 349, 88-91 (2015).

15. Swanstrom, J. A. et al. Dengue virus envelope dimer epitope monoclonal antibodies isolated from dengue patients are protective against Zika virus. $m$ Bio 7, 1123-1131 (2016).

16. Fibriansah, G. et al. A potent anti-dengue human antibody preferentially recognizes the conformation of $\mathrm{E}$ protein monomers assembled on the virus surface. EMBO Mol. Med. 6, 358-371 (2014).

17. Rouvinski, A. et al. Recognition determinants of broadly neutralizing human antibodies against dengue viruses. Nature 520, 109-113 (2015).

18. Fahimi, H., Mohammadipour, M., Haddad Kashani, H., Parvini, F. \& Sadeghizadeh, M. Dengue viruses and promising envelope protein domain IIIbased vaccines. Appl. Micro. Biot. 102, 2977-2996 (2018).

19. Guzman, M. G., Hermida, L., Bernardo, L., Ramirez, R. \& Guillen, G. Domain III of the envelope protein as a dengue vaccine target. Expert Rev. Vaccines $\mathbf{9}$, 137-147 (2010).

20. Qu, P. et al. Insect cell-produced recombinant protein subunit vaccines protect against Zika virus infection. Antivir. Res. 154, 97-103 (2018).

21. Yang, M., Dent, M., Lai, H., Sun, H. \& Chen, Q. Immunization of Zika virus envelope protein domain III induces specific and neutralizing immune responses against Zika virus. Vaccine 35, 4287-4294 (2017).

22. Flipse, J. \& Smit, J. M. The complexity of a dengue vaccine: a review of the human antibody response. PLoS Negl. Trop. Dis. 9, 3749-3767 (2015).

23. Collins, M. H. et al. Human antibody response to Zika targets type-specific quaternary structure epitopes. JCI Insight 4 https://doi.org/10.1172/jci. insight.124588 (2019).

24. Martin, J. \& Hermida, L. Dengue vaccine: an update on recombinant subunit strategies. Acta virologica 60, 3-14 (2016).

25. Luo, Y. Y. et al. Identification of a novel infection-enhancing epitope on dengue prM using a dengue cross-reacting monoclonal antibody. $B M C$ Microbiol. 13, 194 (2013).

26. Baronti, C. et al. Complete coding sequence of zika virus from a French polynesia outbreak in 2013. Genome Announc. 2 https://doi.org/10.1128/ genomeA.00500-14 (2014).

27. Brien, J. D., Lazear, H. M. \& Diamond, M. S. Propagation, quantification, detection, and storage of West Nile virus. Curr. Protoc. Microbiol. 31, 15D 13 11-15D 1318 (2013)

28. Metz, S. W. et al. In vitro assembly and stabilization of dengue and Zika virus envelope protein homo-dimers. Sci. Rep. 7, 4524 (2017).

29. Henchal, E. A., McCown, J. M., Burke, D. S., Seguin, M. C. \& Brandt, W. E. Epitopic analysis of antigenic determinants on the surface of dengue-2 virions using monoclonal antibodies. Am. J. Trop. Med. Hyg. 34, 162-169 (1985).

\section{Acknowledgements}

This work was supported by grants from the NIH-NIAID grants 1-R01-AI107331-01 (PI A. de Silva), U19 AI109784-01 PI J.Ting), US Department of Defense grant W81-XWH18-2-0035 (PI A. de Silva) and the European Union Zika Plan Research Consortium.

\section{Author contributions}

S.W.M. and A.T. performed the majority of experiments. A.B., J.F., and M.J.M. expressed and purified ZIKV monomer and dimer proteins. C.A.L., H.M.L., and S.T. performed the 
animal experiments, S.W.M., S.T., and A.M.dS. designed the study. S.W.M. wrote the paper and all authors reviewed the paper.

\section{Competing interests}

The authors declare no competing interests.

\section{Additional information}

Supplementary information is available for this paper at https://doi.org/10.1038/s41467019-12677-6.

Correspondence and requests for materials should be addressed to S.W.M. or A.M.d.S.

Peer review information Nature Communications thanks the anonymous reviewers for their contribution to the peer review of this work. Peer reviewer reports are available.

Reprints and permission information is available at http://www.nature.com/reprints
Publisher's note Springer Nature remains neutral with regard to jurisdictional claims in published maps and institutional affiliations.

(c) (i) Open Access This article is licensed under a Creative Commons Attribution 4.0 International License, which permits use, sharing, adaptation, distribution and reproduction in any medium or format, as long as you give appropriate credit to the original author(s) and the source, provide a link to the Creative Commons license, and indicate if changes were made. The images or other third party material in this article are included in the article's Creative Commons license, unless indicated otherwise in a credit line to the material. If material is not included in the article's Creative Commons license and your intended use is not permitted by statutory regulation or exceeds the permitted use, you will need to obtain permission directly from the copyright holder. To view a copy of this license, visit http://creativecommons.org/licenses/by/4.0/.

(C) The Author(s) 2019 